\title{
INTERACCIONES MICROBIANAS: EFECTO DE HONGOS BIOCONTROLADORES Y SOLUBILIZADORES DE FÓSFORO EN LOS ESTADIOS PRE-SIMBIÓTICOS DE HONGOS MICORRÍCICO ARBUSCULARES
}

\author{
IVANA F. DELLA MÓNICA ${ }^{1}$, ALICIA M. GODEAS² y JOSÉ M. SCERVINO³
}

\begin{abstract}
Summary: Microbial interactions: effect of biocontrol and $P$ solubilizing fungi in pre-symbiotic stages of arbuscular mycorrhizal fungi. Phosphorus $(P)$ is an essential macronutrient with a low bioavailability in soils, thus limiting the plant growth. The use of $P$ solubilizing fungi $(\mathrm{HS})$ is a promising alternative to increase the efficiency of P-fertilizers. However, it is not fully understood if HS have antagonist effects on beneficial soil microbiota, such as arbuscular mycorrhizal fungi (HMA). This work studies the effect of HS with biocontrol abilities against phytopathogenic fungi on the pre-infective stages development of HMA, the first step in mycorrhizal root colonization. Spores of the HMA Gigaspora rosea were exposed in vitro to different exudate concentrations $(0 ; 0,01 ; 0,1 ; 1 ; 3 \%)$ of different HS fungal strains (Talaromyces helicus strain L7B, T. helicus strain N24, T. diversus and Penicillium purpurogenum) in a system containing semi-liquid medium. Spores germination, elongation, ramification and morphology of the pre-infective mycelium of HMA were evaluated. We observed that only $T$. diversus increased germination while $T$. helicus L7B promoted the elongation, ramification and produced changes in the mycelial morphology without affecting spores' germination. These results showed that germination and pre-infective development of $G$. rosea can be modified in length and ramification as a function of the identity of the HS strain used, suggesting that microbial interactions can positively impact on plant $\mathrm{P}$ nutrition through the modification of the previous stage to mycorrhizal symbiosis.
\end{abstract}

Key words: Arbuscular mycorrhiza, Gigaspora, microbial interactions, P solubilizing fungi, pre-infective, Symbiosis, Talaromyces.

Resumen: El fósforo $(\mathrm{P})$ es un macronutriente poco biodisponible para las plantas y por lo tanto, limitante para su crecimiento. El uso de hongos solubilizadores de $\mathrm{P}(\mathrm{HS})$ es una alternativa prometedora en el aprovechamiento de la fertilización fosforada. Sin embargo, aún no se ha establecido si los HS tienen efectos sobre la micobiota benéfica del suelo, como los hongos micorrícico-arbusculares (HMA). Este trabajo estudia el efecto de HS con capacidad biocontroladora de fitopatógenos fúngicos sobre el desarrollo micorrícico preinfectivo de HMA, el primer paso en la colonización de la raíz, de los HMA. Se expusieron esporas del HMA Gigaspora rosea a distintas concentraciones de exudados $(0 ; 0,01 ; 0,1 ; 1 ; 3 \%)$ de distintos HS: Talaromyces helicus cepa L7B, T. helicus cepa N24, T. diversus y Penicillium purpurogenum, en un sistema in vitro con medio semilíquido. Se evaluó la germinación de las esporas y la elongación, ramificación y morfología del micelio pre-infectivo. Se observó que solo T. diversus incrementó la germinación y que T. helicus L7B promovió la elongación, ramificación y modificó la morfología del micelio pre-infectivo del HMA sin afectar la germinación. Estos resultados demuestran que el desarrollo pre-infectivo de G. rosea (germinación, elongación, ramificación y morfología) puede ser modulado en función de la cepa de HS estudiada, sugiriendo que las interacciones microbianas podrían impactar positivamente sobre la nutrición fosforada vegetal mediante la modificación del estadio previo a la simbiosis micorrícica.

Palabras clave: Gigaspora, hongos solubilizadores de fósforo, interacciones microbianas, micorrizas arbusculares, pre-infectivo, simbiosis, Talaromyces.

\footnotetext{
${ }^{1}$ Instituto de Micología y Botánica (INMIBO-CONICET) - Universidad de Buenos Aires, Facultad de Ciencias Exactas y Naturales, Ciudad Universitaria, Pabellón 2, 4 Piso, Departamento de Biodiversidad y Biología Experimental, laboratorio 69. Buenos Aires, Argentina. e-mail: ivanadm@bg.fcen.uba.ar, ifdellamonica@gmail.com.

2 Instituto de Biodiversidad y Biología Experimental y Aplicada (IBBEA-CONICET) - Universidad de Buenos Aires, Facultad de Ciencias Exactas y Naturales, Ciudad Universitaria, Pabellón 2, $4^{\circ}$ Piso, Departamento de Biodiversidad y Biología Experimental, laboratorio 12. Buenos Aires, Argentina.

${ }^{3}$ Instituto de Investigaciones en Biodiversidad y Medio Ambiente (INIBIOMA-CONICET) - Universidad Nacional de Comahue, San Carlos de Bariloche, Río Negro, Argentina.
} 


\section{INTRODUCCIÓN}

El incremento de la demanda de alimentos a nivel global presenta desafíos a la hora de implementar técnicas productivas, sustentables y amigables con el medio ambiente. El fósforo $(\mathrm{P})$ es un nutriente esencial limitante en el crecimiento y rendimiento de los cultivos. La utilización de bioinoculantes como los hongos solubilizadores de fósforo (HS) para incrementar la disponibilidad de $\mathrm{P}$ para las plantas y su productividad es una herramienta prometedora siendo hasta ahora poco utilizada (Osorno \& Osorio, 2014). Los bioinoculantes en base a estos microorganismos deben aportar un beneficio para los cultivos, sin generar efectos adversos sobre el ecosistema en el cual se aplica, particularmente a la microbiota benéfica asociada a las raíces de las plantas. Los hongos micorrícico arbusculares (HMA), son microorganismos simbiontes radicales presentes en el suelo que, además de tener un importante rol en el transporte del P soluble desde el suelo hacia las plantas, les confieren múltiples beneficios frente a situaciones de estrés como resistencia a sequía, salinidad, metales pesados, entre otros (Nadeem et al., 2014).

En la rizósfera, cohabitan una amplia diversidad de microorganismos entre los que se encuentran los HS y los HMA (Khan et al., 2010). Los HS incrementan la disponibilidad del $\mathrm{P}$ en la solución del suelo, permitiendo que las plantas lo incorporen. Por su parte, los HMA absorben el P soluble del suelo en zonas lejanas a las raíces y lo transfieren hacia las plantas por medio de estructuras intracelulares altamente especializadas para el intercambio de nutrientes (Brundrett, 2004). Numerosos estudios muestran que la aplicación de microorganismos benéficos como HS y/o HMA logran mejorar el crecimiento y productividad de diversos cultivos $(\mathrm{Hu}$ et al., 2009; Della Mónica et al., 2014). La aplicación combinada de bacterias solubilizadoras de $\mathrm{P}$ y HMA en ensayos de invernadero está documentada en varios hospedantes, con resultados positivos (Boostani et al., 2014; Nadeem et al., 2014). Asimismo, otros trabajos muestran sinergismo entre HS y HMA en plantas (Şahin et al., 2004; Khan, 2007). Sin embargo, el conocimiento del efecto que los HS tienen sobre los HMA es escaso, siendo necesario realizar estudios más profundos acerca de las interacciones entre los microorganismos del suelo para poder elegir combinaciones que favorezcan el desarrollo y crecimiento de las plantas.

El establecimiento de la simbiosis micorrícica puede estar influenciada por distintas vías a través de las poblaciones de microorganismos del suelo (Johansson et al., 2004; Scervino et al., 2009; Della Mónica et al., 2015). La etapa de crecimiento presimbiótico en los HMA, al igual que la germinación, puede ser influenciada por distintos factores y es crucial para el establecimiento de la colonización micorrícica (Akiyama \& Hayashi, 2006; Scervino et al., 2007). La presencia de exudados radicales solubles y volátiles, especialmente flavonoides y estrigolactonas, puede llegar a incrementar 8 veces la producción de micelio en esta etapa (Peters \& Verma, 1990; Scervino et al., 2007). Asimismo, sustancias secretadas por microorganismos también pueden modular el desarrollo hifal pre-infectivo (Fracchia et al., 2004, Scervino et al., 2009; Della Mónica et al. 2014). Dado que los HS producen sustancias que podrían promover o inhibir el crecimiento de los HMA (Della Mónica, 2014), es importante evaluar el impacto de la aplicación de HS sobre los HMA.

En este trabajo se estudia el efecto in vitro de hongos con capacidad biocontroladora de fitopatógenos fúngicos y solubilizadora de $\mathrm{P}$ sobre el hongo micorrícico arbuscular Gigaspora rosea con relación a su desarrollo pre-simbiótico, evaluando la germinación de esporas, y el desarrollo del micelio en función a su elongación, ramificación y morfología.

\section{Materiales y Métodos}

Para la realización de los experimentos se utilizaron esporas del HMA Gigaspora rosea Nicolson \& Schenck (BEG9) y cuatro cepas de HS caracterizadas previamente respecto de la capacidad y eficiencia de solubilización de $\mathrm{P}$ y biocontrol (Rodríguez, 2004; Scervino et al., 2010; Della Mónica, 2014; Della Mónica et al., 2018): T. helicus cepa N24 (BAFC 3126), T. helicus cepa L7B (BAFC 3127), T. diversus (BAFC 3128) y Penicillium purpurogenum (BAFC 3303). Se realizó un diseño factorial completo de 4 (HS) x 5 (concentraciones de exudados), con 5 réplicas por tratamiento. Cada parámetro medido (germinación, longitud hifal, número de ápices y morfología del micelio) fue comparado con tratamientos control realizados con medio de cultivo sin exudados 


\section{F. Della Mónica et al. - Hongos solubilizadores de P sobre micorrizas arbusculares}

(concentración 0\%). Se analizaron los datos realizando un análisis de la varianza (ANOVA) de dos factores (Concentraciones de exudados y HS), luego de la verificación del cumplimiento de los supuestos. Cuando el ANOVA arrojó diferencias significativas $(p \leq 0,05)$, se analizaron los contrastes con la prueba de Tukey HSD.

Las esporas de $G$. rosea fueron obtenidas de plantas de trigo cultivadas en maceta durante 6 meses en sustrato inoculado con $G$. rosea bajo condiciones de $16 / 8$ hs de luz/oscuridad, $25 / 18^{\circ} \mathrm{C}$ día/noche. Mediante el método de tamizado húmedo se recolectaron esporas sanas y morfológicamente homogéneas, y se colocaron sobre un papel de filtro humedecido, conservándose para la esterilización superficial mediante el protocolo de Mosse (1962). Las esporas superficialmente esterilizadas se utilizaron inmediatamente para los ensayos.

Los exudados de HS fueron obtenidos siguiendo el método descrito por Fracchia et al. (2004). Luego de 10 días de crecimiento a $25^{\circ} \mathrm{C}$ con agitación a $125 \mathrm{rpm}$, los cultivos líquidos fueron centrifugados $(3000 \mathrm{~g}, 1 \mathrm{~min})$, filtrados $(0,22 \mu \mathrm{m})$ y los sobrenadantes conservados a $-20^{\circ} \mathrm{C}$ hasta su uso.

Se utilizaron cajas de Petri de $50 \mathrm{~mm}$ de diámetro con $10 \mathrm{ml}$ de medio Gel-GRO semilíquido (ICN Biochemicals, Aurora, Ohio, USA. Gel Gro 4\%, buffer MES 0,195\% pH 7). Este medio semilíquido permite el contacto de toda la espora con los exudados de los hongos (Fracchia et al., 2001). El medio fue suplementado con distintos volúmenes de exudado de HS para obtener distintas concentraciones finales $(0 \% ; 0,1 \% ; 0,5 \% ; 1 \%$ y $3 \%$ ). En cada caja se colocaron diez esporas superficialmente esterilizadas y se incubaron en oscuridad, a $25^{\circ} \mathrm{C}$, durante dos semanas. Se calculó el porcentaje de germinación (\% esporas germinadas/esporas totales) en cada tratamiento. En las esporas germinadas se midió la longitud hifal y el número de ápices hifales, promediando los resultados de los parámetros para cada tratamiento. El micelio se cuantificó bajo lupa por el método de la intersección (Brundrett, 1991), previa tinción con azul Tripán $0,05 \%$ en ácido láctico (Phillips \& Hayman, 1970). La morfología del micelio fue calculada como la proporción entre la longitud hifal y el número de ápices presentes en cada espora germinada (Moore, 2002; Della Mónica et al., 2014).

\section{Resultados}

La germinación de esporas de G. rosea se vio afectada por los exudados de algunas de las cepas de HS $(F=10,95 ; p<0.0001)$ independientemente de su concentración $(\mathrm{F}=0,02 ; \mathrm{p}=0,99)$. La interacción entre los factores estudiados no fue significativa $(\mathrm{F}=1,16 ; \mathrm{p}=0,33)$. Particularmente, los exudados de $T$. diversus incrementaron entre un $70 \%$ y $100 \%$ la germinación respecto a los de T. helicus N24, $P$. purpurogenum, T. helicus L7B y al control (Fig. $1 \mathrm{~A})$.

Independientemente de la concentración de exudado utilizada (ausencia de interacción entre los factores, $\mathrm{F}=0,82 ; \mathrm{p}=0,63$ ), el crecimiento de las hifas de las esporas germinadas (longitud hifal) fue modificado por el factor cepa de $\mathrm{HS}(\mathrm{F}=20,57$; $\mathrm{p}<0.0001)$. En este caso, el agregado de exudados de T. helicus L7B triplicó la longitud hifal respecto al control y las demás cepas fúngicas (Fig. 1B). Por otro lado, la concentración de exudados no modificó la longitud hifal $(\mathrm{F}=2,13 ; \mathrm{p}=0,1)$.

$\mathrm{Al}$ analizar el número de ápices del micelio de esporas germinadas, si bien no hubo interacción entre los factores $(\mathrm{F}=1,478 ; \mathrm{p}=0,1516)$, se encontró que con exudados de T. helicus L7B se duplicó el valor obtenido con respecto al control y fue significativamente mayor al número obtenido con los exudados de las otras cepas (Fig. 1C; $F=13,73$; $\mathrm{p}<0.0001)$. El factor concentración de exudados no produjo diferencias significativas para este parámetro $(\mathrm{F}=0,42 ; \mathrm{p}=0,74)$.

La morfología del micelio pre-infectivo de G. rosea (longitud hifal por unidad de ápice) no se vio afectada por la interacción entre los factores concentración de exudados y cepa de HS $(\mathrm{F}=1,5587 ; \mathrm{p}=0,1229)$. Al estudiar los efectos principales de los factores por separado se observó que, independientemente de la concentración, la morfología del micelio varía según la cepa de HS $(\mathrm{F}=14,34 ; \mathrm{p}<0.0001)$. Los exudados de T. helicus L7B incrementaron un $66 \%$ la longitud hifal por ápice respecto del control (Fig. 2A). Por otro lado, la morfología también fue afectada por el factor concentración de exudados $(\mathrm{F}=6,14 ; \mathrm{p}<0.0001)$. Se observó que la morfología del micelio de la espora germinada varía en función de las concentraciones: con $0,5 \%$ se obtienen los valores significativamente más altos, seguido por las concentraciones $0,1 \%$, $1 \%$ y $3 \%$, en orden decreciente (Fig. 2B). 

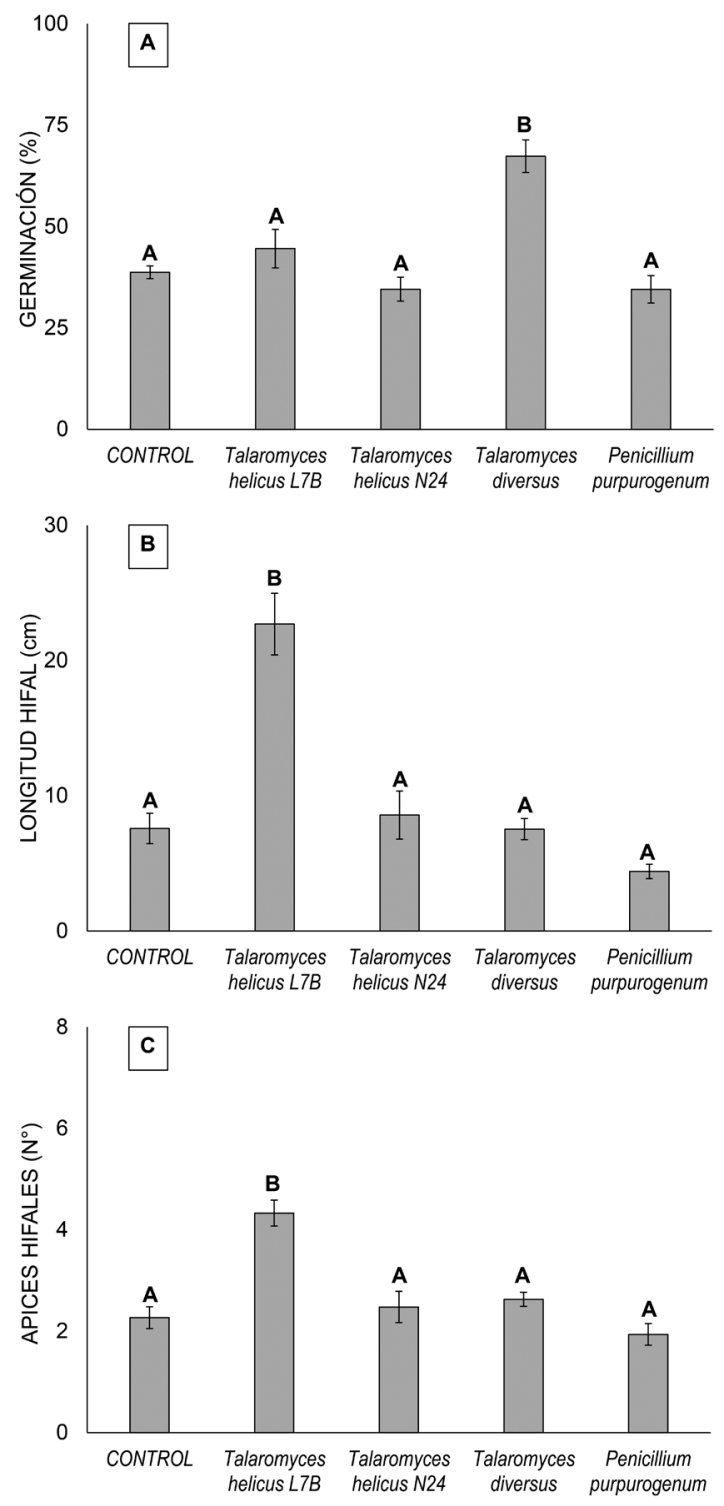

Fig. 1. Desarrollo pre-simbiótico de esporas del hongo micorrícico arbuscular Gigaspora rosea en presencia de exudados de cuatro cepas (Talaromyces helicus cepa L7B, T. helicus cepa N24, Talaromyces diversus y Penicillium purpurogenum) de hongos solubilizadores de $\mathrm{P}$ y biocontroladores de fitopatógenos fúngicos. A: Porcentaje de germinación de esporas. B: Longitud del micelio medida en centímetros. C: Número de ápices hifales presentes en el micelio. Las barras verticales denotan los errores estándar. Distintas letras muestran diferencias estadísticamente significativas según Tukey HSD $(p<0,05)$.

\section{Discusión}

Estudios previos mostraron la importancia del estudio de las interacciones que involucran a los hongos saprobios del suelo y los HMA (Fracchia et al., 2004; Scervino et al., 2008; Scervino et al., 2009; Della Mónica et al., 2014, 2015). Estos microorganismos del suelo pueden influenciar en forma drástica la formación y desarrollo de la simbiosis de HMA, modificando así el efecto sobre el desarrollo de las plantas.
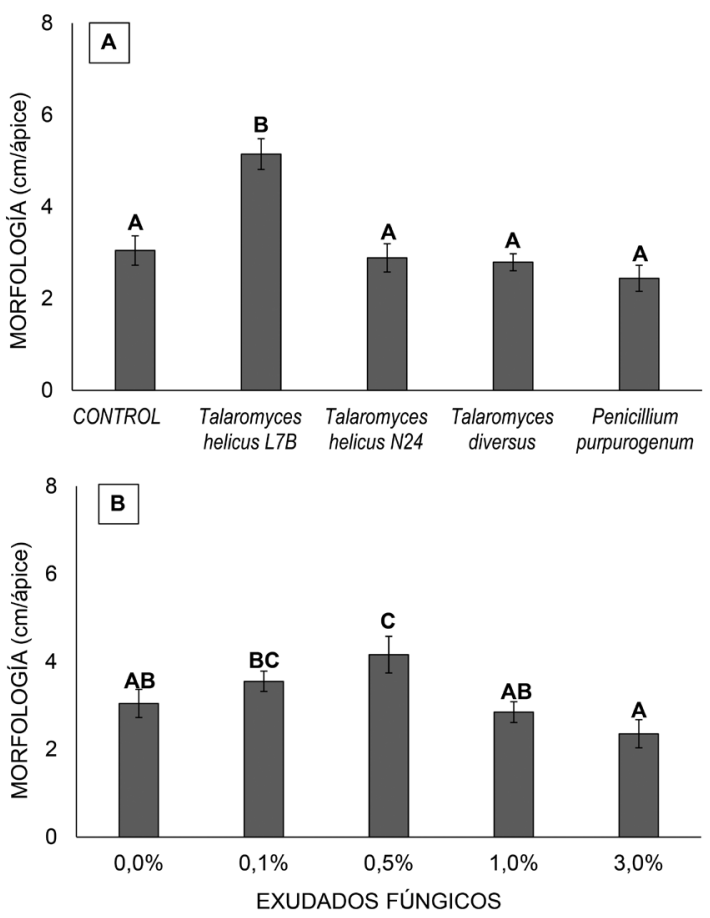

Fig. 2. Efecto de los factores principales (Cepa de hongo solubilizador de $\mathrm{P} \times$ Concentración de exudados) sobre la Morfología del micelio micorrícico de esporas de Gigaspora rosea germinadas, calculado como centímetros de longitud de micelio por unidad de ápice. A: Factor Cepa Fúngica (Talaromyces helicus cepa L7B, T. helicus cepa N24, Talaromyces diversus y Penicillium purpurogenum). B: Factor Concentración de Exudados $(0 \% ; 0,1 \%$; $0,5 \% ; 1 \%$ y $3 \%$ ). Las barras verticales denotan los errores estándar. Distintas letras muestran diferencias estadísticamente significativas según Tukey HSD $(p<0,05)$. 
La germinación de esporas de HMA puede verse afectada por diversos factores como $\mathrm{pH}$, tipo de sustrato, disponibilidad de nutrientes, temperatura, y diversas sustancias como flavonoides, estrigolactonas y/o exudados volátiles y solubles provenientes de plantas y microorganismos (Giovannetti, 2000; Akiyama et al., 2005; Steinkellner et al., 2007). Estudios previos han evaluado el efecto de distintas especies de hongos del suelo sobre la germinación de especies de HMA (Glomus mosseae, Rhizophagus irregularis, Gigaspora margarita) obteniendo resultados variables (Fracchia et al., 1998; Martínez et al., 2004). Fracchia et al. (1998) mostró que Wardomyces inflatus incrementó la germinación y desarrollo de G. mosseae, mientras que Gliocladium roseum, Trichoderma pseudokoningii y Trichoderma harzianum no afectaron estos parámetros. Martínez et al. (2004) mostraron que distintas cepas de T. pseudokoningii inhibieron o tuvieron efectos neutros sobre la germinación de G. mosseae y G. rosea. Otros trabajos han estudiado el efecto de hongos endófitos de vástago, Epichloë, sobre el desarrollo pre-infectivo de HMA observando efectos positivos o neutros sobre la germinación de esporas, la elongación y ramificación del micelio de $G$. rosea y G. margarita, dependiendo de la cepa de Epichloë estudiada (Novas et al., 2011; Vignale et al., 2017). Es importante destacar que estos hongos foliares poseen transmisión vertical, por lo que su colonización es previa a la micorrícica y también pueden modular el patrón de exudación radical, a diferencia de los HS estudiados en este trabajo que no son endofitos y simplemente habitan en la rizósfera y el rizoplano. Si bien se han estudiado los efectos de distintas especies de microorganismos, el estudio de la influencia de HS con capacidad biocontroladora de fitopatógenos fúngicos sobre HMA es incipiente. Talaromyces flavus es una cepa fúngica con capacidad biocontroladora de hongos fitopatógenos (Madi et al., 1997; Tjamos \& Fravel, 1997) y que recientemente se caracterizó como solubilizadora y mineralizadora de P (Della Mónica et al., 2018). Esta cepa incrementó el crecimiento pre-infectivo y la eficiencia micorrícica en plantas de trigo (Della Mónica et al., 2014). Estudios realizados con hongos endófitos septados oscuros, capaces de mineralizar y solubilizar distintas fuentes de $\mathrm{P}$, mostraron que Phaeomollisia piceae interactúa con $G$. rosea incrementando la toma de
P del suelo en trébol (Della Mónica et al., 2015). Nuestros resultados muestran que los exudados de $T$. diversus, incrementan significativamente la germinación de las esporas de G. rosea, mientras que los exudados de T. helicus N24, T. helicus L7B y $P$. purpurogenum no tienen un efecto neto sobre este parámetro. Esto indica una clara dependencia de la especie de HS involucrada en la interacción. Esta dependencia podría deberse a que cada especie libera compuestos de diferente naturaleza química al medio y que la germinación de las esporas de HMA puede ser afectada por los mismos.

Los experimentos realizados en este trabajo muestran que los diferentes HS por medio de sus exudados modifican parámetros pre-infectivos como la longitud hifal de las esporas germinadas de $G$. rosea y el número de ramificaciones hifales que desarrollan. Puntualmente, los exudados de T. helicus L7B, aproximadamente triplicaron el desarrollo hifal y duplicaron el nivel de ramificación con respecto al control, sugiriendo que esta especie de HS produce sustancias solubles que favorecen el desarrollo pre-infectivo del HMA. Sin embargo, la naturaleza de los compuestos involucrados en la modulación del desarrollo micorrícico pre- y post- simbiótico por parte de los microorganismos que cohabitan en la rizósfera es poco conocida. Estudios previos mostraron que ciertos organismos saprótrofos como algunas levaduras en la fase de metabolismo secundario produjeron aumentos sobre el desarrollo hifal y la ramificación del micelio del HMA (Fracchia et al., 2004; Scervino et al., 2008). Es interesante notar que los metabolitos producidos por las distintas especies de hongos no siempre actúan de la misma forma en el desarrollo pre-infectivo de las micorrizas. Un claro ejemplo es $T$. helicus L7B el cual, si bien no modificó la germinación de las esporas, incrementó los parámetros de elongación y ramificación hifal, modificando aún la morfología del micelio; mientras que la cepa $T$. diversus incrementó la germinación, pero no afectó otros parámetros de desarrollo de HMA. El aumento en el crecimiento del micelio pre-infectivo $\mathrm{y} / \mathrm{o}$ en el número de ápices hifales, podría traducirse en una mayor infección sobre las raíces de las plantas (debido al incremento de la posibilidad de contacto entre una mayor cantidad de ápices de HMA y las raíces), e influir positivamente en el transporte de nutrientes. De hecho, Della 
Mónica et al. (2014) mostraron que los exudados producidos por T. flavus incrementaron parámetros en el desarrollo del micelio pre-infectivo de $G$. rosea y este incremento se tradujo en una mayor colonización micorrícica e incorporación de $\mathrm{P}$ en plantas de trigo, sugiriendo que esto también podría ocurrir con otras especies del género Talaromyces. Es interesante notar que las especies estudiadas de Talaromyces fueron caracterizadas previamente como hongos biocontroladores de patógenos vegetales (Madi et al., 1997; Tjamos \& Fravel, 1997; Rodríguez, 2004), lo que podría producir mayores beneficios en su aplicación sobre cultivos en situaciones de estrés biótico, aunque se deben realizar más estudios para comprobar esta afirmación.

El agregado de exudados de T. helicus L7B modificó la morfología del micelio incrementando en un $66 \%$ la elongación por unidad de ápice hifal con respecto al control. Por otro lado, concentraciones de exudados superiores al $1 \%$ produjeron menor elongación hifal por ápice que concentraciones menores. Estudios previos realizados con G. rosea mostraron que compuestos secretados por las raíces (estrigolactonas) estimulan el desarrollo pre-infectivo e inducen cambios en las esporas germinadas, incrementando el número de ramificaciones hifales (Besserer et al., 2006). También se ha mostrado que la morfología del micelio se ve afectada por exudados radicales, incrementando la ramificación de las esporas germinadas hacia una estructura en forma de abanico (del inglés "fan-like structure") (Juge et al., 2009). Por el contrario, una mayor elongación por ápice muestra una mejor capacidad para la exploración del sustrato por parte de la micorriza. Todos estos estudios sumados a los nuestros sugieren que la presencia de estas sustancias en las cercanías de la espora germinada actúa como sensor de la proximidad de las raíces a ser colonizadas. Posiblemente las cepas de HS estudiadas secreten sustancias análogas a las segregadas por las raíces y que desencadenen un cambio en la morfología de HMA maximizando la colonización radical y exploración del sustrato.

El desarrollo pre-infectivo de HMA mostró una amplia gama de respuestas para los parámetros estudiados, desde efectos neutros (T. helicus N24), hasta estimulación (T. helicus L7B) dependiendo de la cepa fúngica analizada. Esto demuestra que los exudados de hongos solubilizadores y biocontroladores estudiados presentan sustancias que son capaces de actuar como moduladores del desarrollo pre-infectivo de los HMA dependiendo de la variabilidad intraespecífica de las cepas. En conjunto, nuestros resultados apoyan la hipótesis que los efectos sobre el desarrollo hifal preinfectivo de los HMA dependen de la naturaleza y concentración de los exudados de los hongos solubilizadores y biocontroladores (Della Mónica, 2014).

El estudio de las interacciones microbianas tiene un alto impacto en el desarrollo y aplicación de tecnologías que mejoren la producción de cultivos. Es importante destacar que los hongos estudiados además de ser biocontroladores de patógenos, son solubilizadores de $\mathrm{P}$, por lo que estos resultados demuestran que la relación existente entre los facilitadores y los transportadores de $\mathrm{P}$ es previa al establecimiento de la simbiosis micorrícica. Asimismo, esta interacción microbiana podría influir en la nutrición fosforada de las plantas mediante el incremento de la disponibilidad de $\mathrm{P}$ soluble por los HS y la modulación del desarrollo pre-infectivo de los HMA.

\section{Agradecimientos}

Agradecemos al Consejo Nacional de Investigaciones Científicas y Técnicas (CONICET) y a la Agencia Nacional de Promoción Científica y Tecnológica (ANPCyT) por el apoyo financiero brindado.

\section{Bibliografía}

AKIYAMA, K. \& H. HAYASHI. 2006. Strigolactones: Chemical signals for fungal symbionts and parasitic weeds in plant roots. Ann. Bot. 97: 925-931.

AKIYAMA, K., K. MATSUZAKI \& H. HAYASHI. 2005. Plant sesquiterpenes induce hyphal branching in arbuscular mycorrhizal fungi. Nature 435: 824827.

BESSERER, A., V. PUECH-PAGÈS, P. KIEFER, V. GOMEZ-ROLDAN, A. JAUNEAU, S. ROY, J. C. PORTAIS, C. ROUX, G. BÉCARD \& N. SÉJALONDELMAS. 2006. Strigolactones stimulate arbuscular mycorrhizal fungi by activating mitochondria. PLoS Biol. 4: e226. 


\section{F. Della Mónica et al. - Hongos solubilizadores de P sobre micorrizas arbusculares}

BOOSTANI, H.R., M. CHOROM, A.A. MOEZZI \& N. ENAYATIZAMIR. 2014. Mechanisms of plant growth promoting rhizobacteria (PGPR) and mycorrhizae fungi to enhancement of plant growth under salinity stress: A review. Sci. J. Biol. Sci. 3: 98-107.

BRUNDRETT, M.C. 2004. Diversity and classification of mycorrhizal associations. Biol. Rev. 79:473-495.

BRUNDRETT, M.C. 1991. Mycorrhizas in natural ecosystems. In: MACFAYDEN, A., M. BEGON \& A.H. FITTER (eds.) Advances in Ecological Research, pp. 171-313, London.

DELLA MÓNICA, I.F., M.S. GODOY, A.M. GODEAS \& J.M. SCERVINO. 2018. Fungal extracellular phosphatases: their role in $\mathrm{P}$ cycling under different $\mathrm{pH}$ and $\mathrm{P}$ sources availability. J. Apll. Microbiol. 124: $155-165$.

DELLA MÓNICA, I.F., M.C.N. SAPARRAT, A. M. GODEAS \& J.M. SCERVINO. 2015. The coexistence between DSE and AMF symbionts affects plant $\mathrm{P}$ pools through $\mathrm{P}$ mineralization and solubilization processes. Fungal Ecol. 17: 10-17.

DELLA MÓNICA, I.F. 2014. Hongos solubilizadores y movilizadores de fósforo en la interfase sueloraíz-planta. Análisis de los mecanismos implicados en su solubilización. Facultad de Ciencias Exactas y Naturales. Universidad de Buenos Aires. Tesis Doctoral, pp. 69-111.

DELLA MÓNICA, I.F., P.J. STEFANONI RUBIO, R.P. CINA, M. RECCHI, A.M. GODEAS \& J.M. SCERVINO. 2014. Effects of the phosphatesolubilizing fungus Talaromyces flavus on the development and efficiency of the Gigaspora rosea-Triticum aestivum symbiosis. Symbiosis 64: 25-32.

FRACCHIA, S., I. SAMPEDRO, J.M. SCERVINO, I. GARCIA ROMERA, A. GODEAS \& J.A. OCAMPO. 2004. Influence of saprobe fungi and its exudates on arbuscular mycorrhizal symbiosis. Symbiosis 36: 169-182.

FRACCHIA, S., A. MENÉNDEZ, A.M. GODEAS \& J.A. OCAMPO. 2001. A method to obtain monosporic cultures of arbuscular mycorrhizal fungi. Soil. Biol. Biochem. 33: 1283-1285.

FRACCHIA, S., M.T. MUJICA, I. GARCIA-ROMERA, J.M. GARCIA-GARRIDO, J. MARTIN, J.A. OCAMPO \& A. GODEAS. 1998. Interactions between Glomus mosseae and arbuscular mycorrhizal sporocarp-associated saprophytic fungi. Plant Soil 200:131-137.

GIOVANNETTI, M. 2000. Spore germination and presymbiotic mycelial growth. In: KAPULNIK, Y. \& D.D DOUDS JR. (eds.) Arbuscular Mycorrhizas: Physiology and function, pp. 47-68. Springer, London.
HU, J., X. LIN, J. WANG, J. DAI, X. CUI, R. CHEN \& J. ZHANG. 2009. Arbuscular mycorrhizal fungus enhances crop yield and P-uptake of maize (Zea mays $L$.): A field case study on a sandy loam soil as affected by long-term P-deficiency fertilization. Soil Biol. Biochem. 41: 2460-2465.

JOHANSSON, J. F., L. R. PAUL \& R. D. FINLAY. 2004. Microbial interactions in the mycorrhizosphere and their significance for sustainable agriculture. FEMS Microbiol Ecol. 48: 1-13.

JugE, C., A. P. COUGHLAN, J. A. FORTÍN \& Y. PICHÉ. 2009. Growht and branching of asymbiotic, presymbiotic, and extraradical AM fungal hyphae: clarification of concepts and terminology. In: KHASA, D., Y. PICHÉ \& A. P. COUGHLAN (eds). Advances in Mycorrhizal Science and Technology, pp. 39-50. NRC Research Press, Ottawa.

KHAN, I. A., S. AHMAD, S. N. MIRZA, M. NIZAMI, M. ATHAR \& S. M. SHABBIR. 2007. Growth response of buffel grass (Cenchrus ciliaris) to phosphorus and mycorrhizal inoculation. Agric. Conspec. Sci. 72: 129-132.

KHAN, M. S., A. ZAIDI, M. AHEMAD, M. OVES \& P. A. WANI. 2010. Plant growth promotion by phosphate solubilizing fungi - current perspective. Arch. Agron. Soil Sci. 56: 73-98.

MADI L., T. KATAN, J. KATAN \& Y. HENIS. 1997. Biological control of Sclerotium rolfsii and Verticillium dahliae by Talaromyces flavus is mediated by different mechanisms. Phytopatol. 87: 1054-1060.

MARTINEZ, A., M. OBERTELlO, A. PARDO, J. A. OCAMPO \& A. GODEAS. 2004. Interactions between Trichoderma pseudokoningii strains and the arbuscular mycorrhizal fungi Glomus mosseae and Gigaspora rosea. Mycorrhiza 14: 79-84.

MOORE, D. 2002. Hyphal growth. In: MOORE, D (ed.), Fungal morphogenesis, pp. 26-63. Cambridge University Press, Cambridge.

MOSSE, B. 1962. The establishment of vesiculararbuscular mycorrhiza under aseptic conditions. $J$. Gen. Microbiol. 27: 509-520.

NADEEM, S. M., M. AHMAD, Z. A. ZAHIR, A. JAVAID \& M. ASHRAF. 2014. The role of mycorrhizae and plant growth promoting rhizobacteria (PGPR) in improving crop productivity under stressful environments. Biotechnol. Adv. 32: 429-448.

NOVAS, M. V., L. J. IANONNE, A. M. GODEAS \& J. M. SCERVINO. 2011. Evidence for leaf endophyte regulation of root symbionts: effect of Neotyphodium endophytes on the pre-infective state of mycorrhizal fungi. Symbiosis 55: 19-28.

OSORNO, L. \& N. W. OSORIO. 2014. Effect of carbon and nitrogen source and concentration on rock phosphate dissolution induced by fungi. J. App. Biotechnol. 2: 32-42. 
PETERS, N. \& D. VERMA. 1990. Phenolic compounds as regulators of gene expression in plant-microbe interactions. Mol. Plant Microbe Interact. 3: 1990.

PHILLIPS, J. M. \& D. S HAYMAN. 1970. Improved procedure for clearing roots and staining parasitic and vesicular-arbuscular fungi for rapid assessment of infection. Trans. Br. Mycol. Soc. 55: 158-161.

RODRÍGUEZ, M. A. 2004. Hongos del suelo antagonistas de Sclerotinia Sclerotiorum: selección y estudio de potenciales agentes de biocontrol. Facultad de Ciencias Exactas y Naturales. Universidad de Buenos Aires. Tesis Doctoral, pp. 153-351.

ŞAHIN, F., R. ÇAKMAKÇI \& F. KANTAR. 2004. Sugar beet and barley yields in relation to inoculation with $\mathrm{N}_{2}$-fixing and phosphate solubilizing bacteria. Plant Soil 265: 123-129.

SCERVINO, J. M., M. P. MESA, I. F. DELLA MÓNICA, M. RECCHI, N. S. MORENO \& A. GODEAS. 2010. Soil fungal isolates produce different organic acid patterns involved in phosphate salts solubilization. Biol. Fertil. Soil. 46: 755-763.

SCERVINO, J. M., A. GOTTLIEB, V. A. SILVANI, M. PÉRGOLA, L. FERNÁNDEZ \& A. M. GODEAS. 2009. Exudates of dark septate endophyte (DSE) modulate the development of the arbuscular mycorrhizal fungus (AMF) Gigaspora rosea. Soil Biol. Biochem. 41: 1753-1756.

SCERVINO, J. M., I. SAMPEDRO, M. A. PONCE, M. A. RODRIGUEZ, J. A. OCAMPO \& A. GODEAS. 2008. Rhodotorulic acid enhances root colonization of tomato plants by arbuscular mycorrhizal (AM) fungi due to its stimulatory effect on the presymbiotic stages of the AM fungi. Soil Biol. Biochem. 40: 2474-2476.
SCERVINO, J. M., M. A. PONCE, R. ERRABASSELLS, J. BOMPADRE, H. VIERHEILIG, J. A. OCAMPO \& A. M. GODEAS. 2007. The effect of flavones and flavonols on colonization of tomato plants by arbuscular mycorrhizal fungi of the genera Gigaspora and Glomus. Can. J. Microbiol. 53: 702709.

STEINKELLNER, S., V. LENDZEMO, I. LANGER, P. SCHWEIGER, T. KHAOSAAD, J. P. TOUSSAINT \& H. VIERHEILIG. 2007. Flavonoids and strigolactones in root exudates as signals in symbiotic and pathogenic plant-fungus interactions. Molecules 12: 1290-1306.

TJAMOS E. C. \& D. R. FRAVEL. 1997. Distribution and establishment of the biocontrol fungus Talaromyces flavus in soiland on roots of solanaceous crops. Crop Prot. 16: 135-139.

VIGNALE, M. V., L. J. IANNONE, J. M. SCERVINO \& M. V. NOVAS. 2017. Epichloë exudates promote in vitro and in vivo arbuscular mycorrhizal fungi development and plant growth. Plant Soil 422: $267-$ 281.

Recibido el 20 de agosto de 2017, aceptado el 25 de abril de 2018. Editor: Leopoldo Iannone. 